University of Nebraska - Lincoln

DigitalCommons@University of Nebraska - Lincoln

Ralph Skomski Publications

Research Papers in Physics and Astronomy

4-15-1997

Superparamagnetic ultrathin films

\author{
Ralph Skomski \\ University of Nebraska-Lincoln, rskomski2@unl.edu \\ D. Sander \\ Max-Planck-Institut für Mikrostrukturphysik \\ J. Shen \\ Max-Planck-Institut für Mikrostrukturphysik, shenj5494@fudan.edu.cn \\ J. Kirschner \\ Max-Planck-Institut für Mikrostrukturphysik
}

Follow this and additional works at: https://digitalcommons.unl.edu/physicsskomski

Part of the Physics Commons

Skomski, Ralph; Sander, D.; Shen, J.; and Kirschner, J., "Superparamagnetic ultrathin films" (1997). Ralph Skomski Publications. 29.

https://digitalcommons.unl.edu/physicsskomski/29

This Article is brought to you for free and open access by the Research Papers in Physics and Astronomy at DigitalCommons@University of Nebraska - Lincoln. It has been accepted for inclusion in Ralph Skomski Publications by an authorized administrator of DigitalCommons@University of Nebraska - Lincoln. 


\title{
Superparamagnetic ultrathin films
}

\author{
R. Skomski, D. Sander, J. Shen, and J. Kirschner \\ Max-Planck-Institut für Mikrostrukturphysik, Weinberg 2, 06120 Halle, Germany
}

The finite-temperature magnetism of ultrathin films such as sesquilayer $\mathrm{Fe} / \mathrm{W}(110)$ and submonolayer $\mathrm{Fe} / \mathrm{Cu}(111)$ is investigated. Based on renormalizations of Onsager's exact solution of the two-dimensional Ising model it is shown that superparamagnetism is a common phenomenon in imperfect ultrathin films. The ultimate reason for this behavior is the existence of two structural length scales: the lattice constant, usually considered in renormalization-group theory, and the characteristic size of the film inhomogeneities. (C) 1997 American Institute of Physics. [S0021-8979(97)47408-X]

\section{INTRODUCTION}

A key problem in the magnetism of ultrathin metallic films is the existence of ferromagnetic order in films with ferromagnetic interatomic coupling. ${ }^{1-4}$ From the point of view of statistical mechanics, ferromagnetism is characterized by a singular Curie temperature $T_{c}$, below which there is long-range ferromagnetic order. In a strict sense, there is no ferromagnetism in nature, since the magnets' finite size inhibits ferromagnetic long-range order in a thermodynamic sense. For example, isotropic nanocrystalline permanent magnets are actually random-anisotropy spin glasses, in spite of their often very high coercivity. ${ }^{5}$

In this theoretical paper we investigate the superparamagnetism of inhomogeneous films such as sesquilayer $\mathrm{Fe} /$ $\mathrm{W}(110)$ consisting of second-layer islands on a monolayer background ${ }^{2,3,6}$ and submonolayer fcc iron on flat and vicinal $\mathrm{Cu}(111)$ surfaces, which form triangles and stripes, respectively. ${ }^{7}$

\section{THEORETICAL BACKGROUND}

\section{A. Ferromagnetism and superparamagnetism}

The equilibrium partition function $Z=\Sigma \exp (-\beta \mathscr{H})$ $=\Sigma \exp \left(-\mathscr{H} \mid k_{B} T\right)$ shows that ferromagnetism is restricted to magnets extending to infinity in each of the relevant dimensions, since the behavior in the vicinity of $T_{c}$ is determined by long-range thermodynamical correlations. ${ }^{8-10} \mathrm{By}$ comparison, the spontaneous magnetization of superparamagnets is zero, although the magnetization is more or less homogeneous inside each superparamagnetic region.

Figure 1 shows typical ferromagnetic, paramagnetic, and superparamagnetic equilibrium magnetization curves. ${ }^{11}$ The number $N$ of atoms belonging to a superparamagnetic region is obtained from the zero-field slope of the hysteresis loops, $N=k_{B} T(\partial M / \partial H) / \mu_{\mathrm{Fe}} \mu_{0} M_{0}$, where $M_{0}$ is the saturation magnetization. $N=1$, which is simple paramagnetism, leads to a very flat magnetization curve whose slope is below the resolution of Fig. 1, whereas $N=\infty$ yields a step function. Macroscopic magnets, where $N$ is very large, have a finite zero-field slope but cannot be distinguished from true ferromagnets. Since it is difficult to resolve fields much smaller than $0.1 \mathrm{mT}$, clusters having less than about $10^{6}$ spins, or $\sim$ $10^{4} \mathrm{~mm}^{3}$ iron, are superparamagnetic. Typical inhomogeneities in ultrathin films, such as submonolayer triangles of fcc iron on $\mathrm{Cu}(111)$, have volumina of the order $50 \mathrm{~nm}^{3}$, which indicate the relevance of superparamagnetic excitations.

\section{B. Superparamagnetic blocking}

In practice it is necessary to distinguish between superparamagnetism in a narrower sense, characterized by the absence of hysteresis, ${ }^{12}$ and frozen or blocked superparamagnetism involving the magnetocrystalline anisotropy $K_{1}$. Consider, for instance, small ferromagnetic particles of volume $V$ whose zero-field magnetization $M=M_{z}$ equals $M_{0}$ at some time $t=0$. Due to thermal excitations involving the energy barrier $K_{1} V$ this initial magnetization decays after some blocking or relaxation time. ${ }^{11}$

The relaxation times vary between microseconds for ultrasmall iron particles and many millions of years for small inclusions of iron oxides in rocks. ${ }^{11,13}$ However, the time scale is the only difference between blocked and unblocked superparamagnetism, whereas the spontaneous magnetization of a ferromagnet does not decay within any finite time. ${ }^{14}$ In this sense, hysteresis is neither a necessary nor a sufficient condition for ferromagnetism.

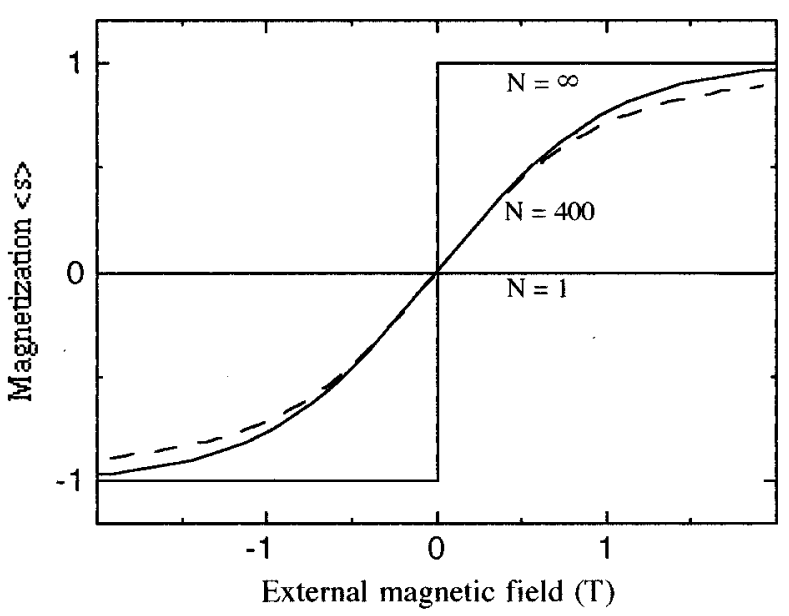

FIG. 1. Ferromagnetic $(N=\infty)$, superparamagnetic $(N=400)$, and paramagnetic $(N=1)$ magnetization curves at $T=270 \mathrm{~K}$. The dashed line refers to an interacting Ising-spin chain. 


\section{The Ising model}

The Ising model is defined by the Hamiltonian $\mathscr{H}$ $=\sum_{i>k} J_{i k} s_{i} s_{k}-h \Sigma_{i} s_{i}$ where $s_{i}= \pm 1$ and $h=N \mu_{B} \mu_{0} H .{ }^{10}$ Usually one assumes that the exchange constants obey $J_{i k}$ $=J$ for nearest neighbors and $J_{i k}=0$, but this restriction does not affect the qualitative behavior of the magnet so long as the range of exchange interaction remains finite. ${ }^{9}$

A key feature of the Ising model is the absence of excitations perpendicular to the anisotropy axis. The anisotropy energy per atom reaches at most a few $\mathrm{MJ} / \mathrm{m}^{3}$, corresponding to excitation temperatures of the order $1 \mathrm{~K}$, which speaks in favor of a Heisenberg description. Only in the immediate vicinity of $T_{c}$ does the anisotropy yield Ising behavior by breaking the continuous symmetry of the Heisenberg model. ${ }^{1,15}$ However, anisotropy energies of superparamagnets are proportional to the cluster size $N$, so that superparamagnetic thin-film clusters characterized by uniaxial anisotropies of order $1 \mathrm{MJ} / \mathrm{m}^{3}$ can be regarded as Ising magnets at all temperatures. For Langevin-like magnetization curves in nonuniaxial thin-film superparamagnets see Ref. 4.

The mean-field Curie temperature of the Ising model equals $z J / k_{B}$, where $z$ is the number of nearest neighbors. In reality, the Curie temperature of one-dimensional Ising spin chains $(z=2)$ is zero. ${ }^{9,10}$ As found by Onsager, ${ }^{16}$ the effect of long-range fluctuations is less pronounced in twodimensional Ising magnets. For a square lattice $(z=4)$ the spontaneous magnetization is given.

The mean-field Curie temperature of the Ising model equals $z J / k_{B}$, where $z$ is the number of nearest neighbors. In fact, critical fluctuations yield $T_{c}=0$ for one-dimensional Ising spin chains $(z=2),{ }^{9,10}$ whereas the effect of fluctuations is less pronounced in two dimensions (Onsager). ${ }^{16}$ For a square lattice $(z=4)$ the spontaneous magnetization is given by

$$
M_{s}=M_{0} \sqrt[8]{1-\frac{1}{\sinh ^{4}\left(J / 2 k_{B} T\right)}},
$$

so that $k_{B} T_{c} / J=2 / \ln (1+\sqrt{2})=2.269$. For triangular $(z=6)$ and hexagonal $(z=3)$ lattices, $k_{B} T_{c} / J$ equals $2 / \ln \sqrt{3}=3.641$ and $2 / \ln (2+\sqrt{3})=1.519$, respectively. ${ }^{9}$ If the interatomic coupling is known, then the formulas for the square and triangular lattices yield rough estimates for the Curie temperatures of bcc(110) and fcc(111) monolayers, respectively.

\section{MAGNETISM OF ULTRATHIN FILMS}

\section{A. Sesquilayer iron on $\mathrm{W}(110)$}

Sesquilayer $\mathrm{Fe} / \mathrm{W}(110)$ films, having nominal thicknesses between one and two monolayers, consist of secondlayer islands on a monolayer background. ${ }^{3}$ Let us describe the ferromagnetic coupling in the monolayer and secondlayer regions by $J_{1}=4 J$ and $J_{2}=z_{\text {eff }} J$, respectively. To estimate the Curier temperature of the inhomogeneous film we overestimate $J_{2}$ by putting $z_{\text {eff }}=\infty$. For the square model Fig. 2 a single renormalization step, rather than a renormalization-group calculation, is sufficient to determine $T_{c}$. The block-spin transformation yields $J_{\text {eff }}=2 J$, so that
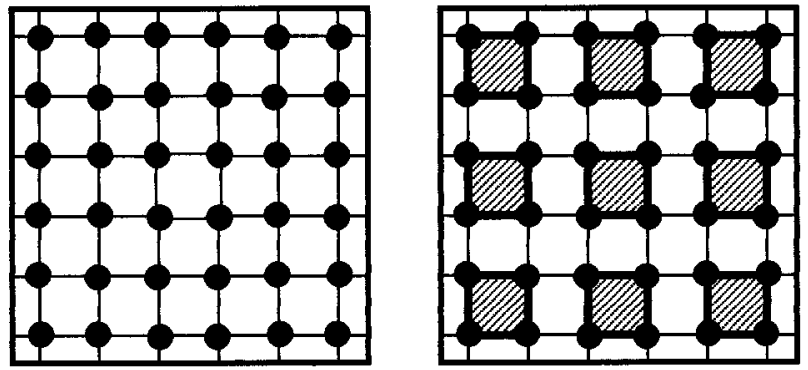

FIG. 2. Schematic structure of monolayer and sesquilayer square lattices. The dashed area shows the second-layer regions.

$$
T_{c}=\frac{4 J}{k_{B} \ln (1+\sqrt{2})} .
$$

Except the different Curie temperature, the magnetization curve is that of the Onsager solution in this particular model (Fig. 3). Note (i) that the bimodal exchange yields a common $T_{c}$ rather than a superposition of two Curie temperatures and (ii) that the Curie temperature is only slightly enhanced by the strong coupling inside the second-layer regions.

Above $T_{c}$ the sesquilayer film is superparamagnetic, since $z_{\text {eff }}=\infty$ assures a perfect spin alignment inside the islands. If $z_{\text {eff }}$ is finite then the superparamagnetism is restricted to a small temperature window between $T_{c}$ and $z_{\text {eff }} J / k_{B}$. Taking $T_{c}=300 \mathrm{~K}$ and $z_{\text {eff }}=6$ this implies superparamagnetism up to about $450 \mathrm{~K}$ for $\mathrm{Fe}(110)$ sesquilayers.

\section{B. Submonolayer fcc iron on $\mathrm{Cu}(111)$}

The deposition of submonolayer iron films on flat and vicinal $\mathrm{Cu}(111)$ surfaces leads to triangular iron islands and stripes oriented along the ledges, respectively. ${ }^{7}$ The behavior of the triangles characteristic of flat surfaces is trivial. Below the blocking temperature $T_{B}$, the triangles are superparamagnetic with a nonnegligible coercivity, and just above $T_{B}$ they are superparamagnets in a narrower sense.

The one-dimensional character of infinitely long stripes means that their Curie temperature is zero. An illustrative proof, which is not restricted to Ising magnets, relies on overestimating $T_{c}$ by dividing the stripe into square blocks

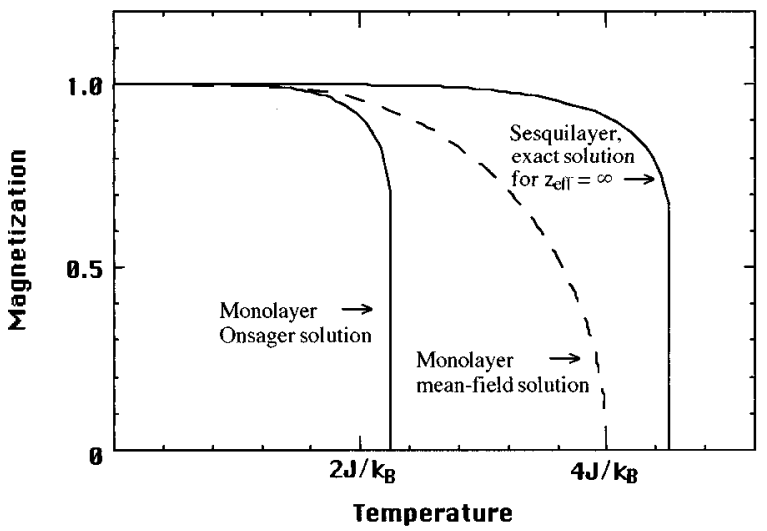

FIG. 3. Temperature dependence of the spontaneous magnetization for the models shown in Fig. 2. 


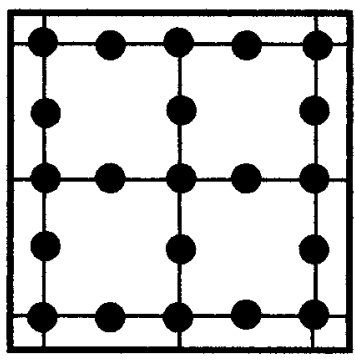

$\mathrm{n}=1$

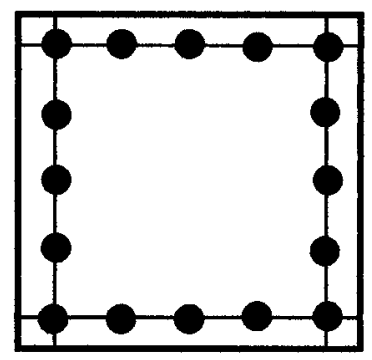

$n=3$
FIG. 4. Two-dimensional networks of Ising chains

inside which the exchange coupling goes to infinity. The coupling between the blocks in then of order $\left(w / R_{\mathrm{Fe}}\right) J$, where $w$ is the width of the stripe. The Curie temperature of the stripe is therefore at most $w / R_{\mathrm{Fe}}$ times the Curie temperature of a simple spin chain, that is $T_{c}=0$ unless $w=\infty$. The claim $^{2}$ that the Curie temperatures of stripes is finite and increases with $w$ is incompatible with the findings of statistical mechanics. A possible explanation is that the stripes percolate and form two-dimensional networks in the sense of this section.

To study the case where the stripes form a percolating two-dimensional network we use the model Fig. 4. $T_{c}$ is determined by removing half of the degrees of freedom by the renormalization transformation

$$
\begin{gathered}
\sum s_{i= \pm 1} \exp \left(\beta J s_{i-1} s_{i}+\beta J s_{i} s_{i+1}\right) \\
=\text { const. } \times \exp \left(\beta J^{\prime} s_{i-1} s_{i+1}\right),
\end{gathered}
$$

where $2 \beta J^{\prime}=\ln \operatorname{ch}(2 \beta J)$. This transformation is well known in the context of one-dimensional magnetism, where it yields $T_{c}=0$. For $n=1$ we obtain after one renormalization step

$$
T_{c}=\frac{2 J}{k_{B} \operatorname{arcosh}(1+\sqrt{2})}=1.308 J / k_{B} .
$$

For long chains

$$
T_{c}=\frac{2 J}{k_{B}[\ln (1+\sqrt{2})+\ln (n+1)]} .
$$

Thus, the Curie temperature of percolating stripes is nonzero and weakly decreases with increasing chain length $n$. For instance, taking $n=100$ yields a Curie temperature reduction by a factor 6.2 compared to an ideal $n=0$ monolayer.

\section{Nonequilibrium behavior}

Since the Ising model has no inherent dynamics, it is necessary to introudce mechanisms such as Glauber transitions ${ }^{14,17}$ defined by the transition rate

$$
W\left(s_{i} \rightarrow-s_{i}\right)=\frac{\Gamma_{0}}{2}\left[1-s_{i} \tanh \left(\frac{h+J s_{i-1}+J s_{i+1}}{k_{B} T}\right)\right] .
$$

Here $\Gamma_{0}=1 / \tau_{0}=\Gamma_{m} / \exp \left(K_{1} V / k_{B} T\right)$, where $\Gamma_{m} \approx 10^{9-11} \mathrm{~s}^{-1}$ is some microscopic attempt frequency. ${ }^{18}$ Equation (6) yields $^{14,17}$

$$
\frac{d\left\langle s_{i}\right\rangle}{d t}=-\Gamma_{0}\left\langle s_{i}\right\rangle+\frac{\Gamma_{0}}{2} \tanh (2 \beta J)\left(\left\langle s_{i-1}\right\rangle+\left\langle s_{i+1}\right\rangle\right),
$$

and the relation $\Gamma=\Gamma_{0}[1-\tanh (2 \beta J) \cos (k a)]$. The relaxation of the average magnetization is given by $\Gamma(k=0)$, and in the limits of zero and very large coupling $J$ the blocking temperatures are $K_{1} V / k_{B} \ln \left(\Gamma_{m} t_{0}\right)$ and $\left(K_{1} V+4 J\right) /$ $k_{B} \ln \left(\Gamma_{m} t_{0}\right)$, respectively. ${ }^{7}$ Here $t_{0} \approx 100 \mathrm{~s}$ is the time necessary to conduct a typical experiment.

\section{DISCUSSION AND CONCLUSIONS}

The models used in this paper have the advantage of yielding exact results. Magnetostatic interactions and incoherent magnetization processes inside the superparamagnetic units are neglected, but due to the inherent weakness of magnetostatic interactions in ultrathin films this assumption is reasonable. A more subtle issue is that the exchange interaction $J$ between the superparamagnetic units and the relaxation rate $\Gamma_{0}$ are only approximately known for real films. This point is irrelevant to the qualitative behavior of the magnet but makes it difficult to compare the predictions of the theory with experimental data. ${ }^{7}$

In conclusion, we have shown that Ising superparamagnetism is common in many ultrathin films. Examples are sesquilayers of $\alpha$ iron on $\mathrm{W}(110)$, where the second-layer islands are superparamagnetic just above the common Curie temperature, and ultrathin stripes and triangles of $\gamma$ iron on $\mathrm{Cu}(111)$ below the Curie temperature of the ideal monolayer reference film. The superparamagnetism considered here is disregarded in the Onsager and renormalization-group theories, where there is only one structural length, namely the lattice constant.

${ }^{1}$ M. Bander and D. L. Mills, Phys. Rev. B 38, 12015 (1988).

${ }^{2}$ H. J. Elmers, J. Hauschild, H. Höche, U. Gradmann, H. Bethge, D. Heuer, and U. Köhler, Phys. Rev. Lett. 73, 898 (1994).

${ }^{3}$ D. Sander, R. Skomski, A. Enders, C. Schmidthals, and J. Kirschner, Phys. Rev. Lett. 77, 2566 (1996).

${ }^{4}$ Y. Park, S. Adenwalla, G. P. Felcher, and S. D. Bader, Phys. Rev. B 52, 12779 (1995).

${ }^{5}$ R. Skomski, J. Magn. Magn. Mater. (in press).

${ }^{6}$ U. Gradmann, Dreikönigstreffen Bad Honnef, 1996 (unpublished); U. Krey, ibid.

${ }^{7}$ For experimental evidence based on STM micrographs and Kerr hysteresis loops, see J. Shen et al., these proceedings.

${ }^{8}$ L. P. Kadanoff, W. Götze, D. Hamblen, R. Hecht, E. A. S. Lewis, V. V. Palciauskas, M. Rayl, J. Swift, D. Aspnes, and J. Kane, Rev. Mod. Phys. 39, 395 (1967).

${ }^{9}$ D. C. Mattis, The Theory of Magnetism II: Thermodynamics and Statistical Mechanics (Springer, Berlin, 1985).

${ }^{10}$ J. M. Yeomans, Statistical Mechanics of Phase Tansitions (Oxford University Press, Oxford, 1992).

${ }^{11}$ L. Néel, Ann. Géophys. (France) 5, 99 (1949).

${ }^{12}$ E. Kneller, Ferromagnetismus (Springer, Berlin, 1962).

${ }^{13}$ R. Skomski and V. Christoph, Phys. Status Solidi B 156, K149 (1989).

${ }^{14}$ K.-H. Fischer and A. J. Hertz, Spin Glasses (Cambridge University Press, Cambridge, 1991).

${ }^{15}$ C. H. Back, C. Würsch, and D. Pescia, Z. Phys. B 98, 69 (1995).

${ }^{16}$ L. Onsager, Phys. Rev. 65, 117 (1944)

${ }^{17}$ A. J. Bray, Adv. Phys. 43, 357 (1994).

${ }^{18}$ P. Gaunt, J. Appl. Phys. 59, 4129 (1986). 